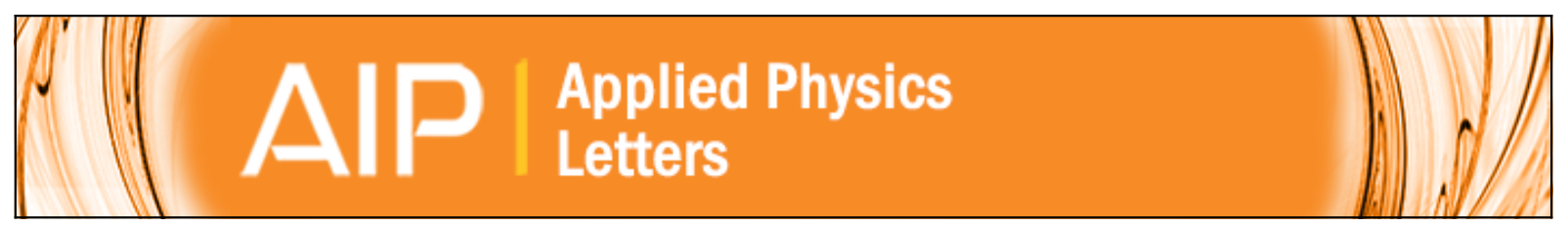

\title{
All-dielectric periodic terajet waveguide using an array of coupled cuboids
}

I. V. Minin, O. V. Minin, V. Pacheco-Peña, and M. Beruete

Citation: Applied Physics Letters 106, 254102 (2015); doi: 10.1063/1.4923186

View online: http://dx.doi.org/10.1063/1.4923186

View Table of Contents: http://scitation.aip.org/content/aip/journal/apl/106/25?ver=pdfcov

Published by the AIP Publishing

\section{Articles you may be interested in}

Terajets produced by dielectric cuboids

Appl. Phys. Lett. 105, 084102 (2014); 10.1063/1.4894243

Dielectric waveguide vertically coupled to all-silicon photodiodes operating at telecommunication wavelengths

Appl. Phys. Lett. 102, 171106 (2013); 10.1063/1.4803541

Periodically focused modes in chains of dielectric spheres

Appl. Phys. Lett. 100, 061123 (2012); 10.1063/1.3684246

Optical waveguide fabrication in z -cut lithium niobate ( LiNbO 3 ) using femtosecond pulses in the low repetition rate regime

Appl. Phys. Lett. 88, 111109 (2006); 10.1063/1.2186389

Waveguide fabrication in phosphate glasses using femtosecond laser pulses

Appl. Phys. Lett. 82, 2371 (2003); 10.1063/1.1565708
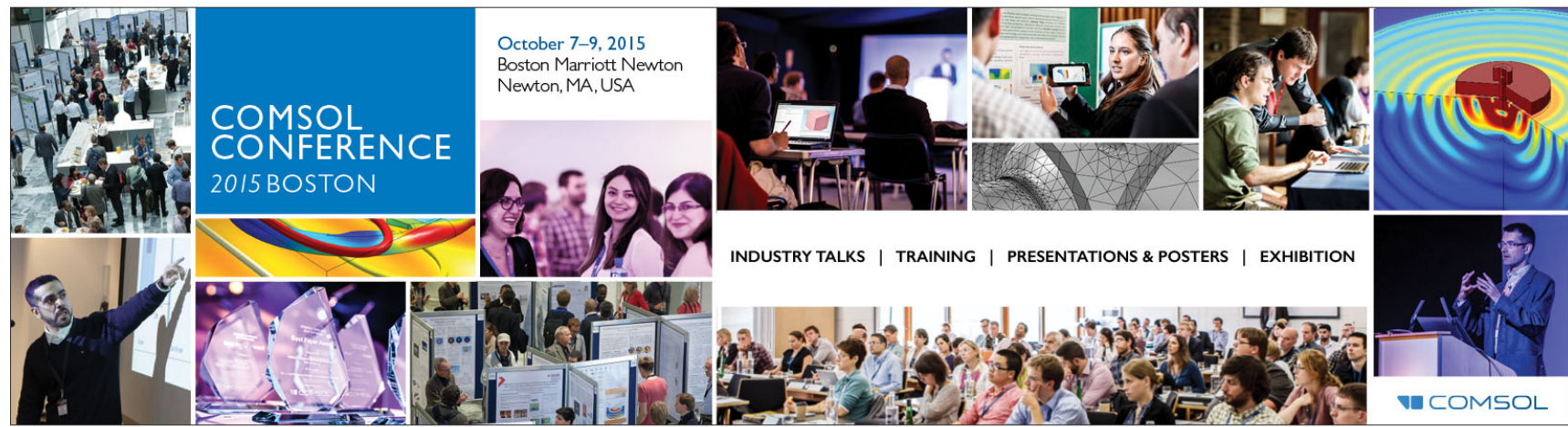


\title{
All-dielectric periodic terajet waveguide using an array of coupled cuboids
}

\author{
I. V. Minin,, ${ }^{1, a)}$ O. V. Minin, ${ }^{1, b)}$ V. Pacheco-Peña, ${ }^{2, c)}$ and M. Beruete ${ }^{2, d)}$ \\ ${ }^{1}$ Siberian State Institute of Metrology, Dimitrova 4, Novosibirsk 630004, Russia \\ ${ }^{2}$ Antennas group - TERALAB, Universidad Pública de Navarra, Campus Arrosadía, 31006 Pamplona, Spain
}

(Received 1 May 2015; accepted 17 June 2015; published online 24 June 2015)

\begin{abstract}
In this paper, the recently proposed technique to produce photonic jets (terajets at terahertz $(\mathrm{THz})$ frequencies) using 3D dielectric cuboids is applied in the design of a mesoscale cuboid-chain waveguide. The chains are basically designed with several dielectric cubes with side $\lambda_{0}$ placed periodically along the axial z-axis and separated by an air-gap. Based on this, a systematic study of the focusing properties and wave guiding of this chain is performed when the air-gap between the dielectric cubes is changed from $0.25 \lambda_{0}$ to $3 \lambda_{0}$ with the best performance achieved at $2.5 \lambda_{0}$. An analysis when losses are included in the cubes is also done, demonstrating a robust performance. Finally, the wave guiding is experimentally demonstrated at sub- $\mathrm{THz}$ frequencies with a good agreement with numerical results. The simulation results of focusing and transport properties are carried out using Finite Integration Technique. The results here presented may be scaled to any frequency ranges such as millimeter, sub-millimeter, and optical frequencies. (C) 2015 AIP Publishing LLC. [http://dx.doi.org/10.1063/1.4923186]
\end{abstract}

Probably, the first experimental prototype of a lens beam waveguide with a set of identical lenses was designed by Christian and Goubau in 1961 for a wavelength of $1.25 \mathrm{~cm}$ and clearly demonstrated its potential. ${ }^{1}$ In this system, several dielectric lenses with diameter of $20 \mathrm{~cm}$ made of expanded polystyrene were used periodically spaced by $1 \mathrm{~m}$. To reduce absorption losses in lens materials, the diffractive lens ${ }^{2}$ was first used in quasi-optical transmission lines by Sobel et al. in $1961 .^{3}$ It was demonstrated that the transmission line had low losses at $210 \mathrm{GHz}$ (only $2 \mathrm{~dB}$ over a distance of $17 \mathrm{~m}$ ).

As it is well known, common lens transmission lines are designed by using lenses with thickness much smaller than its diameter. Based on this, several types of lens transmission lines have been reported using dielectric lenses. ${ }^{4}$ On the other hand, at optical frequencies, it has been also demonstrated that linear arrays of dielectric spheres can operate as lossless waveguides for beams with certain spatial properties which depend on the relative size of the dielectric spheres. ${ }^{5}$

The periodically focused modes (PFMs) concept has been stimulated by studies of chains designed with mesoscale spheres (i.e., diameters of particles of $4 \lambda_{0}<\mathrm{D}<10 \lambda_{0}$, where $\lambda_{0}$ is the working wavelength) ${ }^{6}$ and cylinders. ${ }^{7}$ These studies have been performed in the context of coupling between their whispering gallery modes due to the beam focusing produced by individual spheres and micro-disks when they are illuminated with a planewave, i.e., photonic jets or nanojets. ${ }^{8-11}$ This can be achieved due to the fact that the Brewster angle conditions are periodically reproduced for PFMs with a period matching the size of two sphere diameters, and the coupling between nanojets in chains of spheres. ${ }^{12}$ The latter mechanism leads to the formation of the so-called periodic nanojet-induced modes observed in chains

\footnotetext{
${ }^{a)}$ Electronic mail: prof.minin@gmail.com

b)Electronic mail: prof.minin@gmail.com

${ }^{c)}$ Electronic mail: victor.pacheco@unavarra.es

d)Electronic mail: miguel.beruete@unavarra.es
}

of polystyrene microspheres ${ }^{13-17}$ and cylinders and clusters ${ }^{18}$ of polystyrene microspheres, in which the nanojets can be periodically reproduced along a chain of microspheres. These modes result from the optical coupling of microspheres acting as a series of micro-lenses, which periodically focus propagating waves into photonic nanojets. It has been demonstrated $^{19}$ that the coupled focused beams decrease in size along the chain of polystyrene microspheres with index $n=1.59$, reaching wavelength scale dimensions in the case of small beads with $4<D / \lambda_{0}<10$. It must be noted that all dielectric spheres in such linear arrays are located close to each other and the elementary "unit-cell" of the array consists of two spheres.

Cubic dielectric particles are promising candidates for low-loss, ultra-compact, photonic applications owing to their relative ease of fabrication as compared to spherical particles in integrated optics. By using these structures, the capability to produce jets at terahertz $(\mathrm{THz})$ and sub- $\mathrm{THz}$ frequencies (so-called terajets, analogue of photonic jet in optics) has been recently proposed and demonstrated experimentally by using 2D and 3D dielectric cuboids. ${ }^{20,21}$

In this communication, the capability to produce periodically induced terajets along a chain of mesoscale 3D dielectric cubes (here, at difference with our previous works we use cubes instead of cuboids) separated with air-gaps of different length is numerically studied. Numerical results demonstrate that the optimum air-gap length is $2.5 \lambda_{0}$. Moreover, the influence of dielectric losses in the wave guiding performance is evaluated by introducing overestimated values of loss tangent $(\tan \delta)$, demonstrating that the structure here proposed has a robust performance, due to the fact that the spatial resolution of the terajets produced at the output surface of each cube of the chain is not strongly deteriorated when losses are increased. Finally, the wave guiding using this structure is experimentally demonstrated at sub$\mathrm{THz}$ frequencies using a chain of 5 cubes, with a good agreement with numerical results. The results here presented may 
(a)

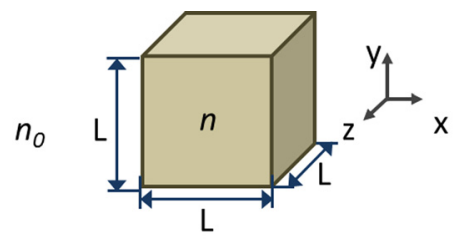

(b)

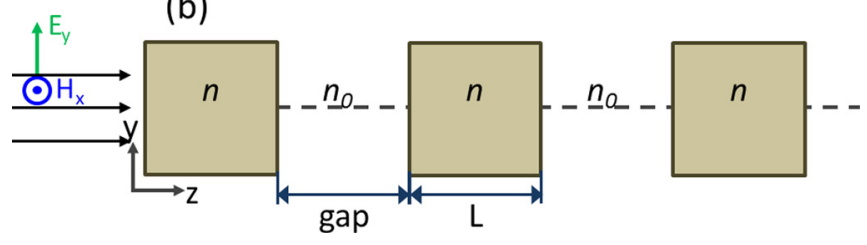

FIG. 1. (a) Schematic representation of a $3 \mathrm{D}$ dielectric cube with side $L=\lambda_{0}$. The $3 \mathrm{D}$ cube has a refractive index $n=1.46$ and is immersed in vacuum $\left(n_{o}=1\right)$. (b) Schematic representation of a chain of $3 \mathrm{D}$ dielectric cubes immersed in vacuum with an air-gap between them.

be directly scaled to other frequency bands such as optical frequencies. All the numerical results were performed using the Finite Integration Technique with the transient solver of the commercial software CST Microwave Studio ${ }^{\mathrm{TM}}$ with the same boundary conditions and mesh as in Refs. 20-22.

The $3 \mathrm{D}$ elementary dielectric cube used in this letter is schematically shown in Fig. 1(a) with side $L=\lambda_{0}$, refractive index $n=1.46$, and immersed in vacuum $\left(n_{0}=1\right)$. In order to
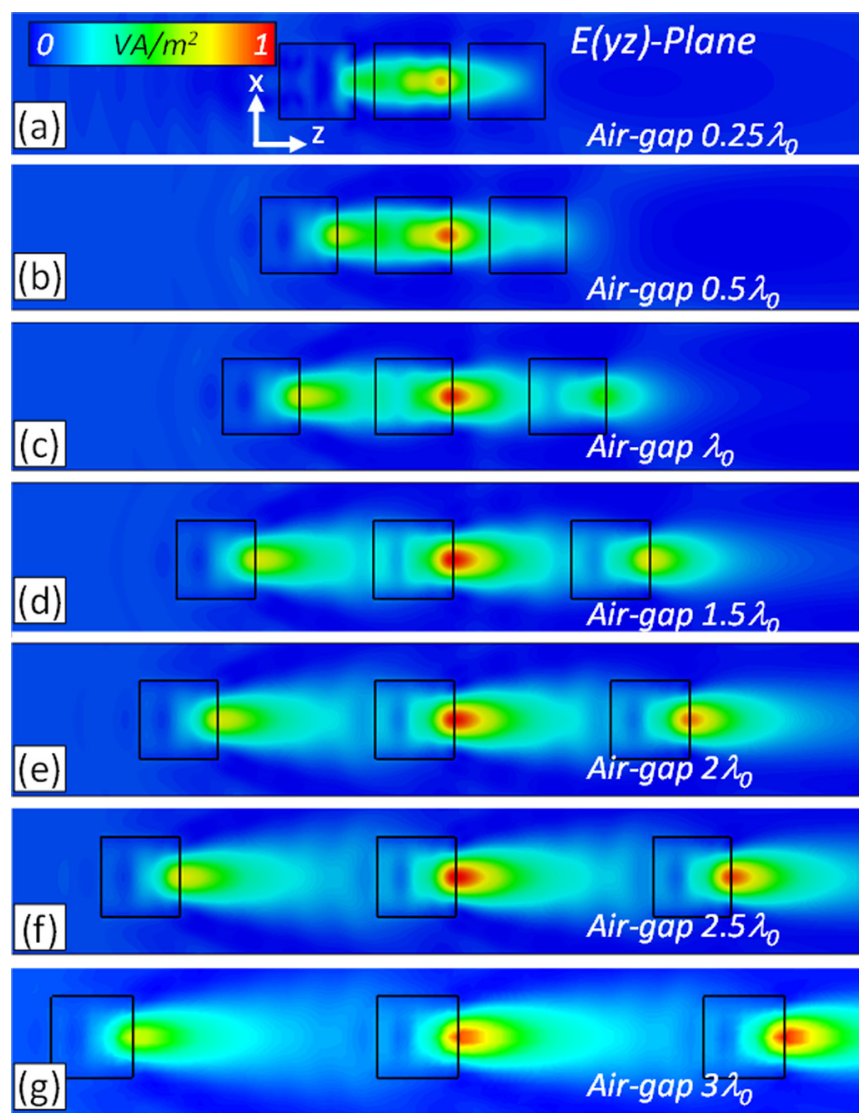

FIG. 2. Numerical simulations of the power distribution on the $y z$-plane/Eplane for several chains of 3D dielectric cuboids with side $L=\lambda_{0}$ when the air-gap between each cuboid is selected as (a) $0.25 \lambda_{0}$, (b) $0.5 \lambda_{0}$, (c) $\lambda_{0}$, (d) $1.5 \lambda_{0}$, (e) $2 \lambda_{0}$, (f) $2.5 \lambda_{0}$, and (g) $3 \lambda_{0}$. The numerical results have been normalized with respect to the maximum power distribution achieved from all the structures which in this case corresponds to the design with an air-gap of $2.5 \lambda_{0}$.
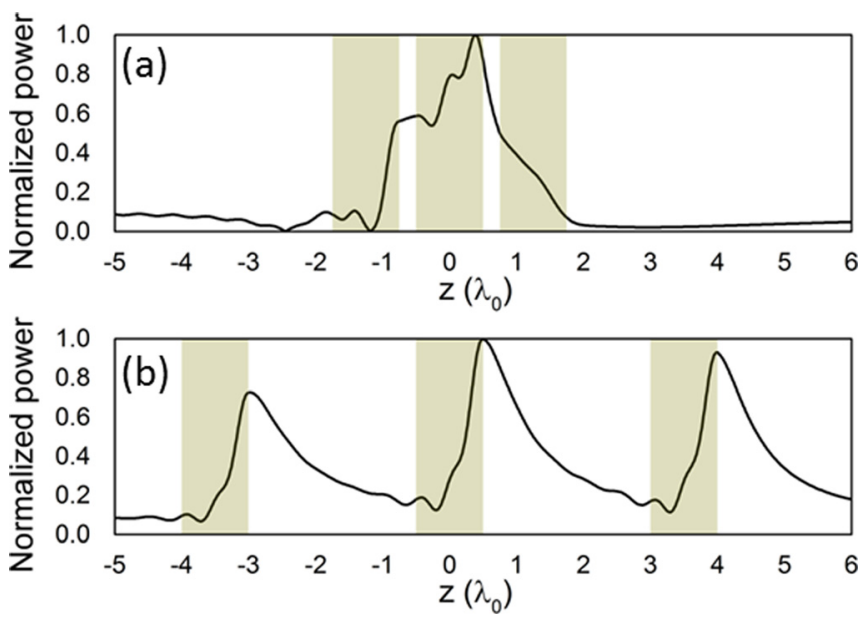

FIG. 3. Simulation results of the normalized power distribution along the optical $z$-axis for two chains of 3D dielectric cuboids shown in Fig. 2, when the air-gap between each cuboid is selected as (a) $0.25 \lambda_{0}$ and (b) $2.5 \lambda_{0}$. Both results are normalized with respect to the maximum power of each case.

design a chain, several cubes (three in this case) are placed periodically along the optical z-axis separated by an air-gap, as shown in Fig. 1(b). The whole structure is illuminated from the left side by using a vertically polarized plane wave $\left(E_{y}\right)$ with its propagation direction along the optical $z$-axis.

Based on this, we first evaluate the focusing performance and wave guiding properties of the chain when the distance between cubes is changed. Numerical results of the normalized power distribution on the E-plane ( $y z$-plane) for values of the air-gaps between cubes varying from $0.25 \lambda_{0}$ to $3 \lambda_{0}$ are shown in Fig. 2 . When the air-gaps are smaller than $\lambda_{0}$, the terajets are produced inside the cubes [Figs. 2(a)-2(c)] and the higher power distribution is obtained at the central cube (close to its output surface). On the other hand, when the air-gaps increase, terajets are clearly formed just at the output surface of each cube. Note that the best performance is achieved when an air-gap of $2.5 \lambda_{0}$ is used, where the power distribution of the terajets is increased for all the cubes [see Fig. 2(f)] while it is reduced when increasing this gap [see Fig. 2(g) for a gap of $3 \lambda_{0}$ ]. In order to better compare the performance, simulation results of the normalized power distribution along the optical $\mathrm{z}$-axis for air-gap values equal to $0.25 \lambda_{0}$ and $2.5 \lambda_{0}$ [taken from Figs. 2(a) and 2(f)] are shown in Fig. 3. Here, it is clearly observed that the best performance is achieved when the air-gap is $2.5 \lambda_{0}$, where the maxima is obtained just at the output surface of each cube, demonstrating that the terajets are efficiently excited with this configuration. It is important to highlight that several simulations with air-gaps up to $4.5 \lambda_{0}$ were performed and the terajets were also observed just at the output of each cube (not shown here). As it has been pointed out previously, the power distribution has a maximum value at the central cube compared with the first one. This is due to the fact that reflection losses are partially compensated by the flat surface of the cubes at the back and front sides. Also, note that the air-gap has an important role in the performance of the chain. This is due to its value should be, at least, the same as the length of the terajet in order to couple one terajet to the next one. This is indicative of the formation of terajet-induced modes having the smallest propagation losses in chains of 


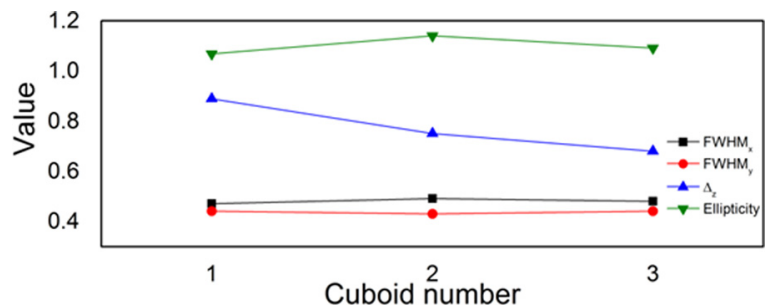

FIG. 4. Numerical results (in wavelengths $\lambda_{0}$ ) of the full-width at half maximum along the $x$ - (black curve) and $y$-axes (red curve) at each output surface of each cuboid. Exploration range in wavelengths $\left(\Delta_{\mathrm{z}}\right)$ for each terajet produced at the output surface of each cuboid (blue curve) along with the ellipticity for each terajet (red curve). All curves have been connected with a continuous line to guide the eye.

cubes. This performance is degraded for smaller gaps since the terajets are not completely formed in such small air-gaps and, therefore, it is not able to couple to the next cube.

For the sake of completeness, numerical results of the full-width at half-maximum (FWHM) along both transversal $x$ - and $y$-axes at each terajet (i.e., at the output surface of each dielectric cube) for the case with air gap $2.5 \lambda_{0}$ are shown in Fig. 4. Here, the cube numbers 1, 2, and 3 correspond to the left, central, and right cube of the chain. It can be observed that a similar resolution is achieved for all the terajets along both directions with values below $0.55 \lambda_{0}$ for all cases. Based on this, the ellipticity (defined as the ratio between both transversal resolutions $\left.\mathrm{FWHM}_{\mathrm{x}} / \mathrm{FWHM}_{\mathrm{y}}\right)^{22}$ of all terajets is close to 1 . Therefore, a quasi-spherical spot is obtained for the chain configuration in good agreement with our previous results dealing with isolated cuboids. ${ }^{20-22}$ Regarding the exploration range $\Delta z$ (defined as the distance from the output surface at which the intensity enhancement has decayed to half its maximum value), ${ }^{20,21}$ it can be observed that the maximum value is obtained for the first cube while it is reduced for the rest. For this chain, the power enhancement (defined as the power at the output surface of each cube compared with the power without cubes) is $\sim 8.5$, $\sim 11.6$, and $\sim 10.8$ (in linear scale) for the first, second, and third cube, respectively, in good agreement with the results of a single cuboid. ${ }^{20}$ Thus, it is observed that a mesoscale dielectric cube-air-chain waveguide is mainly characterized by the periodicity of jets corresponding to the optimal length of air-gap between cubes when a fixed geometry and materials (refractive index contrast between the background medium and the cubes ${ }^{20}$ ) of the cubes is chosen. This fact contrasts with the results found in optical chains of dielectric microspheres where the periodical focusing of light in straight chains of touching microspheres is characterized with the periodicity of photonic nanojets corresponding to the size of two spheres. ${ }^{15}$

Also, it is important to evaluate the performance of the cube-air chain by taking into account the dielectric losses due to the absorption of dielectric materials at $\mathrm{THz}$ frequencies. Numerical results of the normalized power distribution on the $E(y z)$-plane when periodicity is $2.5 \lambda_{0}$ and the cubes have a loss tangent of $\tan \delta=0.05$ and $\tan \delta=0.1$ at the working wavelength $\lambda_{0}$ are shown in Figs. 5(a) and 5(b), respectively. Note that these values are overestimated since, for example, a typical value $\tan \delta$ for Teflon (with a similar refractive index as the one here used) is $\sim 0.008$ at $1 \mathrm{THz} .{ }^{23}$ To better compare
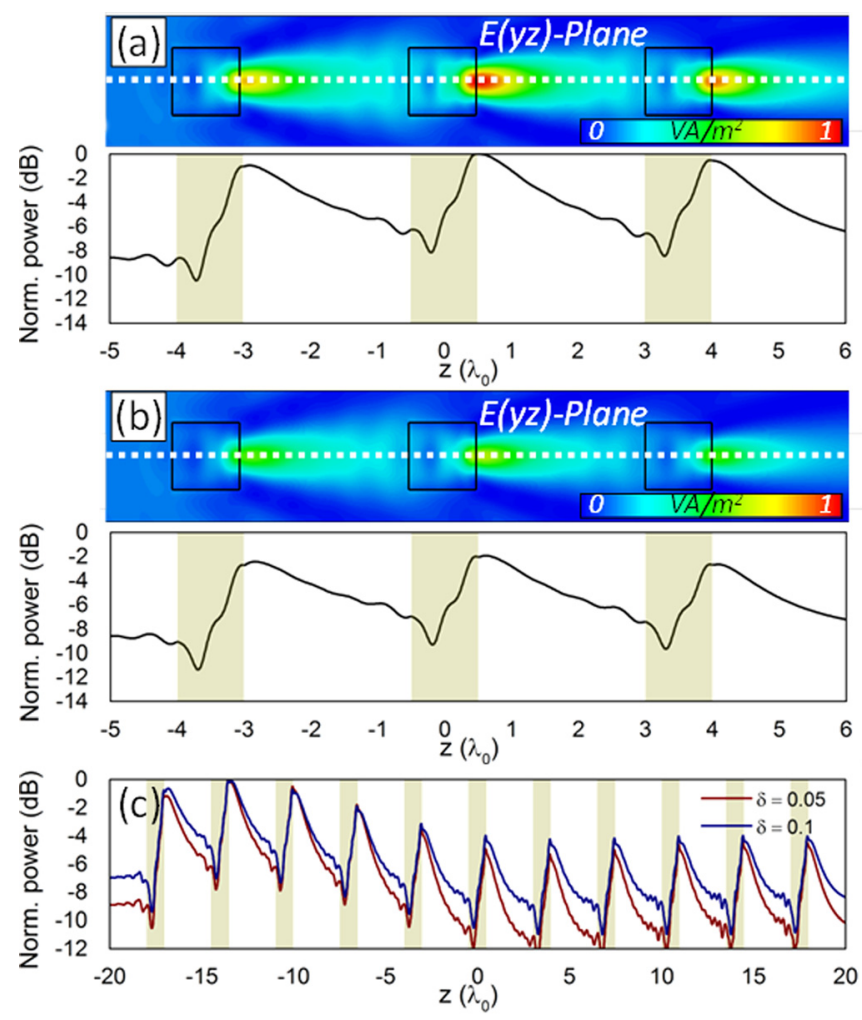

FIG. 5. Numerical simulations of the power distribution on the $E(y z)$-plane a chain of 3D dielectric cuboids with side $L=\lambda_{0}$ with an air-gap between each cuboid with a value of $2.5 \lambda_{0}$ when dielectric losses are introduced to the cuboids with a value of: (a) $\tan \delta=0.05$ and (b) $\tan \delta=0.1$. The bottom plot of each panel represents the power distribution along the optical $z$-axis along the dashed white line for each case. The normalized values are calculated using the maximum from all cases, which corresponds to the terajet produced at the output surface of the second (central) cuboid with $\delta=0.05$. (c) Normalized power distribution along the optical $z$-axis for a chain of 11 dielectric cuboids with the same dimensions as (a) and (b) for the two values of loss tangent $\tan \delta=0.05$ (red line) and $\tan \delta=0.1$ (blue line). The normalized values are calculated based on the maximum of each case.

these results, the power distribution along the optical $z$-axis at $x=0$ is shown at the bottom of each plot for both cases. It can be observed that the terajets are elongated and slightly shifted away from the output surface of each cube when losses are introduced. Moreover, the power distribution at each output is reduced up to $\sim 1 / 3$ when $\tan \delta=0.1$ (compared with the value obtained when no losses are included). These results are not surprising since a change in $\tan \delta$ modifies the refractive index of the cubes and therefore a shift of the focus should occur. ${ }^{20}$ Also the length of terajet is increased when increasing the loss tangent. To better compare these results, the focusing properties of the terajets produced at the output surface of each cube for these values of $\tan \delta$ are summarized in Table I. In general, it is shown that the resolution of each terajet is deteriorated when losses are increased. However, the results obtained are very close to the lossless cases even when the dielectric losses here evaluated are overestimated. For the sake of completeness, the normalized power distribution along the $\mathrm{z}$-axis for two chains of 11 cuboids with the previous values of $\tan \delta$ is shown in Fig. 5(c). It can be observed that the power decays exponentially at half its maximum at the fifth cuboid. Moreover, it is shown that, from the fifth cuboid, the relative power at each terajet still decays but with small losses 
TABLE I. Numerical results of the performance for each terajet produced in a cube-air-chain with an air gap of $2.5 \lambda_{0}$ for different values of loss tangent tan $\delta$.

\begin{tabular}{|c|c|c|c|c|c|c|}
\hline \multirow[b]{2}{*}{ Cube number } & \multicolumn{2}{|c|}{$\mathrm{FWHM}_{\mathrm{x}}\left(\lambda_{0}\right)^{\mathrm{a}}$} & \multicolumn{2}{|c|}{$\operatorname{FWHM}_{\mathrm{y}}\left(\lambda_{0}\right)^{\mathrm{b}}$} & \multicolumn{2}{|c|}{$\Delta_{\mathrm{z}}\left(\lambda_{0}\right)^{\mathrm{c}}$} \\
\hline & $\tan \delta=0.05$ & $\tan \delta=0.1$ & $\tan \delta=0.05$ & $\tan \delta=0.1$ & $\tan \delta=0.05$ & $\tan \delta=0.1$ \\
\hline 1 & 0.49 & 0.5 & 0.45 & 0.47 & 1.1 & 1.37 \\
\hline 2 & 0.5 & 0.51 & 0.43 & 0.45 & 0.97 & 1.71 \\
\hline 3 & 0.49 & 0.5 & 0.43 & 0.44 & 0.83 & 1.01 \\
\hline
\end{tabular}

${ }^{\mathrm{a}} \mathrm{FWHM}_{\mathrm{x}}$ is the full-width at half-maximum along the $x$-axis.

${ }^{\mathrm{b}} \mathrm{FWHM}_{\mathrm{y}}$ is the full-width at half-maximum along the $y$-axis.

${ }^{\mathrm{c}} \Delta_{\mathrm{z}}$ is the photonic jet exploration range.

( $\sim 0.26 \mathrm{~dB} /$ cuboid and $\sim 0.4 \mathrm{~dB} /$ cuboid for the cases with $\tan \delta=0.1$ and $\tan \delta=0.05$, respectively), compared with the first five terajets, and the terajets are excited in the rest of the cuboids which form the chain for both values of $\tan \delta$. It is important to note that these values have been calculated from the normalized power distribution shown in Fig. 5(c), which is normalized to the maximum from each case. Therefore, even when the decay of power at each terajet position is lower for the case with $\tan \delta=0.1$, the total power at the terajet maxima is lower than those obtained with $\tan \delta=0.05$, as it has been explained before and can be corroborated in the bottom panel of Figs. 5(a) and 5(b). The ratio of the power maximum for the cases with $\tan \delta=0.05$ and $\tan \delta=0.1$ is $\sim 1.9 \mathrm{~dB}$. Finally, regarding the FWHM along the transversal $x$-axis for the first, fifth, and last cuboid of Fig. 5(c), the resulting values are $0.49 \lambda_{0}, 0.48 \lambda_{0}$, and $0.49 \lambda_{0}$, respectively, for the case with $\tan \delta=0.05$; while they are $0.5 \lambda_{0}, 0.49 \lambda_{0}$, and $0.5 \lambda_{0}$, respectively, for the case with $\tan \delta=0.1$. This demonstrates that the spatial resolution of the terajets is maintained along both chains. Based on these results, the robustness of the wave guiding configuration here proposed when increasing losses is therefore demonstrated.

Finally, the structure here proposed was evaluated experimentally at sub-THz frequency, specifically at $f_{\text {oexp }}=35 \mathrm{GHz}$ $\left(\lambda_{\text {oexp }}=8.57 \mathrm{~mm}\right)$. For this case, the chain consists of 5 dielectric cubes of Teflon $(n=1.465, \tan \delta=0.00041 \pm 0.00002)$ with side $L=\lambda_{\text {exp }}$ separated by an air-gap of $2.5 \lambda_{\text {oexp }}$. A dielectric platform with a refractive index of 1.02 was used to hold the chain. Moreover, a rectangular horn antenna was used as a source and placed far from the chain in order to illuminate the structure with a planewave. ${ }^{21}$ Also, an absorbing layer was placed surrounding the back side of the first cube to avoid spurious effects due to diffraction. Finally, the power distribution at the output of the chain at $z=0.1 \lambda_{\text {oexp }}$ from the 5th cuboid was recorded using a metallic sphere and measuring its scattered field when it was inserted within the jet and moved along the transversal $x$-axis. ${ }^{2}$ This scattered field was recorded via a rectangular horn antenna used as a receiver and placed at $\sim 3 \lambda_{\text {Oexp }}$ from the sphere [see Figure 6 (a) for the experimental setup]. For the simulation results, the transient solver of the software CST Microwave Studio was employed and the system was modeled as in the experiment, using an absorbing layer for the first cube. The field distribution at the output was obtained by using an electric field frequency monitor at the working frequency.

The experimental and numerical results are shown in Figure 6(b). Here, two cases are shown: all the cubes aligned along the optical $z$-axis (black curves) and last cube shifted horizontally at $x=2 \lambda_{\text {oexp }}$ (red curves). A good agreement between both simulation and experimental results is observed. Moreover, wave guiding is demonstrated with this configuration since, when the 5th cube is moved from the optical axis (red curves), the measured power greatly decays compared with the case when all the cubes are aligned. This is as expected due to the measured power in this case is mainly due to the influence of the 4th cube. Hence, by moving the 5 th cube, the wave guiding is achieved up to the 4 th cube.

In conclusion, the capability to design cube-air-chains waveguides using 3D dielectric cubes of refractive index $n=1.46$ immersed in vacuum $\left(n_{0}=1\right)$ has been studied. Basically, the waveguide has been designed with a periodic series of 3D dielectric cubes with an air-gap between the cubes. A systematic study of the wave guiding performance has been shown by changing the air-gap between the cubes from $0.25 \lambda_{0}$ up to $3 \lambda_{0}$ using three cubes along the optical $z$-axis. This wave guiding stems from the quasi-optical coupling of mesoscale cubes acting as a series of flat lenses,
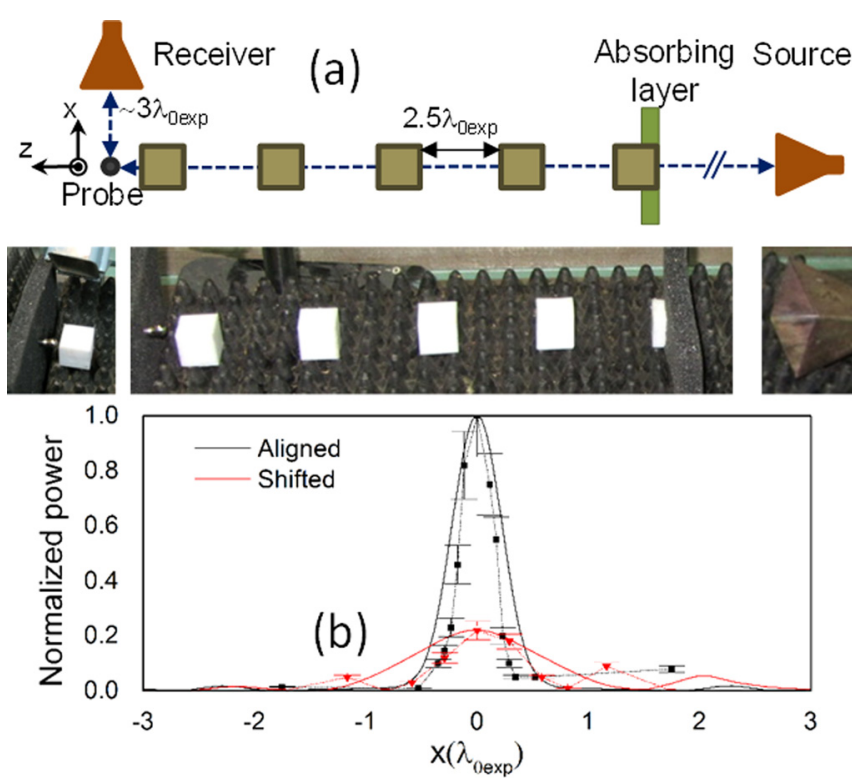

FIG. 6. (a) Schematic representation of the experimental setup (top) along with the pictures of each part of the whole setup (bottom). (b) Simulation (continuous lines) and experimental (dotted lines) results of the power distribution at $z=0.1 \lambda_{\text {oexp }}$. from the last cube of a chain made of 5 cubes separated for an air gap of $2.5 \lambda_{\text {oexp }}$ for the case when all the cubes are aligned along the optical $z$-axis (black curves) and for the case when the last cube (5th) is shifted at $x=2.5 \lambda_{\text {oexp }}$. The experimental error $(15 \%)$ is also shown as error bars for each case. 
which periodically are able to focus the propagating waves generating terajets at their output faces. Based on this, it has been shown that the terajets are not produced for airgaps $<\lambda_{0}$; therefore, no wave guiding is achievable using these configurations. However, by increasing the air-gap, the terajets are generated at the output surface of each cube with an optimum value of air-gap of $2.5 \lambda_{0}$. The focusing properties of the terajets produced at the output surface of each cube of the system have been studied in terms of FWHM along both transversal $x$ - and $y$-axes, terajet exploration range and enhancement. Numerical results demonstrate that values of FWHM below $0.5 \lambda_{0}$ are achievable for all the cubes along both transversal directions. Therefore, a quasispherical terajet is produced with this configuration due to the ellipticity is very small (close to 1). Also, it has been demonstrated that the power enhancement achieved with this design (using three dielectric cubes) is maximum for the terajet produced at the central cube of the chain with a value of $\sim 11$.6. Also, the influence of dielectric losses has been evaluated in terms of both transversal resolution and exploration range by introducing two overestimated values of loss tangent $(\tan \delta=0.05$ and $\tan \delta=0.1)$. It has been shown that, even when higher dielectric losses are introduced in the system, the resolution of the terajets is not strongly deteriorated demonstrating the robustness of the wave guiding configuration here proposed. Finally, this wave guiding has been demonstrated at sub- $\mathrm{THz}$ frequencies and compared with numerical results, demonstrating a very good agreement between both results. Since all the studies here presented have been performed using normalized dimensions for the whole structures, the wave guiding using periodic arranged mesoscale cubes with air gaps may be scaled to other frequency bands such as millimeter, sub-millimeter, and optical frequencies and may be applied to microscopy applications and devices such as power dividers and multiplexers.
This work was supported in part by the Spanish Government under Contract No. TEC2011-28664-C02-01. V.P.-P. was sponsored by Spanish Ministerio de Educación, Cultura y Deporte under Grant No. FPU AP-2012-3796. M.B. was sponsored by the Spanish Government via RYC2011-08221.

${ }^{1}$ G. Christian and J. Goubau, IRE Trans. Antennas Propag. 9, 256 (1961).

${ }^{2}$ I. V. Minin and O. V. Minin, Diffractional Optics of Millimetre Waves, $1 \mathrm{st}$ ed. (The Institute of Physics Publishing, Bristol, UK, 2004).

${ }^{3}$ F. Sobel, F. L. Wentworth, and J. C. Wiltse, IRE Trans. Microwave Theory Tech. 9, 512 (1961).

${ }^{4}$ L. B. Knyaz'kov and N. V. Ruzhentsev, Tech. Phys. Lett. 34, 888 (2008).

${ }^{5}$ A. Darafsheh and V. N. Astratov, Appl. Phys. Lett. 100, 061123 (2012).

${ }^{6}$ V. N. Astratov, J. P. Franchak, and S. P. Ashili, Appl. Phys. Lett. 85, 5508 (2004).

${ }^{7}$ S. Deng, W. Cai, and V. Astratov, Opt. Express 12, 6468 (2004).

${ }^{8}$ Z. Chen, A. Taflove, and V. Backman, Opt. Express 12, 1214 (2004).

${ }^{9}$ P. Ferrand, J. Wenger, A. Devilez, M. Pianta, B. Stout, N. Bonod, E. Popov, and H. Rigneault, Opt. Express 16, 6930 (2008).

${ }^{10}$ A. Heifetz, S.-C. Kong, A. V. Sahakian, A. Taflove, and V. Backman, J. Comput. Theor. Nanosci. 6, 1979 (2009).

${ }^{11}$ D. McCloskey, J. J. Wang, and J. F. Donegan, Opt. Express 20, 128 (2012).

${ }^{12}$ Z. Chen, A. Taflove, and V. Backman, Opt. Lett. 31, 389 (2006).

${ }^{13}$ A. M. Kapitonov and V. N. Astratov, Opt. Lett. 32, 409 (2007).

${ }^{14}$ S. Yang and V. N. Astratov, Appl. Phys. Lett. 92, 261111 (2008).

${ }^{15}$ T. Mitsui, Y. Wakayama, T. Onodera, T. Hayashi, N. Ikeda, Y. Sugimoto, T. Takamasu, and H. Oikawa, Adv. Mater. 22, 3022 (2010).

${ }^{16}$ T. Mitsui, T. Onodera, Y. Wakayama, T. Hayashi, N. Ikeda, Y. Sugimoto, T. Takamasu, and H. Oikawa, Opt. Express 19, 22258 (2011).

${ }^{17}$ O. Lecarme, T. Pinedo Rivera, L. Arbez, T. Honegger, K. Berton, and D. Peyrade, J. Vac. Sci. Technol., B 28, C6O11 (2010).

${ }^{18}$ M. Gerlach, Y. P. Rakovich, and J. F. Donegan, Opt. Express 15, 17343 (2007).

${ }^{19}$ K. W. Allen, A. Darafsheh, and V. N. Astratov, in International Conference on Transparent Optical Networks (2011), p. 2.

${ }^{20}$ V. Pacheco-Peña, M. Beruete, I. V. Minin, and O. V. Minin, Appl. Phys. Lett. 105, 084102 (2014).

${ }^{21}$ V. Pacheco-Peña, M. Beruete, I. V. Minin, and O. V. Minin, Opt. Lett. 40, 245 (2015).

${ }^{22}$ I. V. Minin, O. V. Minin, V. Pacheco-Peña, and M. Beruete, Opt. Lett. 40, 2329 (2015)

${ }^{23}$ Y. Jin, G. Kim, and S. Jeon, J. Korean Phys. Soc. 49, 513 (2006). 